\title{
CLOUD-TO-BIM-TO-VR: AN OPERATIVE APPROACH FOR THE CONSTRUCTION OF INTERACTIVE AND INFORMATION MODELS FOR THE CULTURAL HERITAGE
}

\author{
M. Pulcrano \\ DIARC - Department of Architecture, University of Naples “Federico II”, Italy, margherita.pulcrano@unina.it
}

Commission II

KEY WORDS: Virtual Reality, Cardboard, H-BIM, Point Cloud, Reality-based model, Unity 3D, Game Engine

\begin{abstract}
:
This paper describes the results of a research that aimed to focus on a possible approach in the knowledge, representation and dissemination of the Cultural Heritage through CLOUD-to-BIM-to-VR application processes. Starting from reality-based surveys of the current state and information modelling in BIM platforms, the experimentation conducted involved the construction of a VR fruition system for the Simón Ruiz Hospital in Medina del Campo (Valladolid, Spain). An application for mobile devices was therefore designed to describe the artifact and the information acquired - or processed - during the various research phases according to alternative methods and completely independent from the software that generated them. The fruition system is completed with cardboard type supports, for stereoscopic viewing, and controller, to allow the user to navigate in the represented scene and activate the information hotspots that implement the contents of the App.

Specifically, the results presented here relate to the operational sequences and products obtained in the organization and programming of the App, structured to allow a multidimensional and multimodal fruition path. The characteristics that connote it, therefore, are related to the setting of incremental levels of knowledge, different by type of content displayed - historical, descriptive, technical, etc. -, form in which it is presented - spherical and photographic images, point cloud, geometric and informative model, textual descriptions, etc. - and integration of hotspots for extend the informative and descriptive content.
\end{abstract}

\section{INTRODUCTION}

The renewed interest in the application of virtualization techniques to the Cultural Heritage $(\mathrm{CH})$ and, in particular, the attention paid in recent decades to the integration of the various forms of Extended-Reality (XR) use with the paradigms of information modelling represent the starting point for the experimentation described in this paper.

The simulation of reality through the XR experience, as known, is configured as a promoter of the communication and dissemination of the $\mathrm{CH}$, because it is a tool that reveals the numerous values which connote it (intrinsic/extrinsic, tangible/intangible, material/immaterial, tec.). At the same time, it also plays a mediating role between reality and the (re)proposed digital one, where the user and the built work are connected through new, alternative and (perhaps) unexpected relationships. And to date, these digital connections become even more relevant in the use of the $\mathrm{CH}$ due to the possibility of stemming the critical issues related to the temporary inaccessibility of places - strongly influenced by the pandemic situation that is being experienced - and, consequently, overcoming the different difficulties that affected the whole sector.

Regardless of the fruition's type to be achieved, it is often necessary to virtualize the real space in order to obtain support for the construction of the same; in fact, whether we intervene with AR forms of communication or with VR projects, the digital clone assists in the correct registration and overlap of the contents and/or represents the 3D space being navigated. Clearly the transposition of reality into digital/virtual environments involves careful reflections in the choice of the operative technique to be repeated, in relation to the objectives to be aimed at, because there are many solutions that can be adopted. In this application field, the use of modelling processes and the BIM methodology

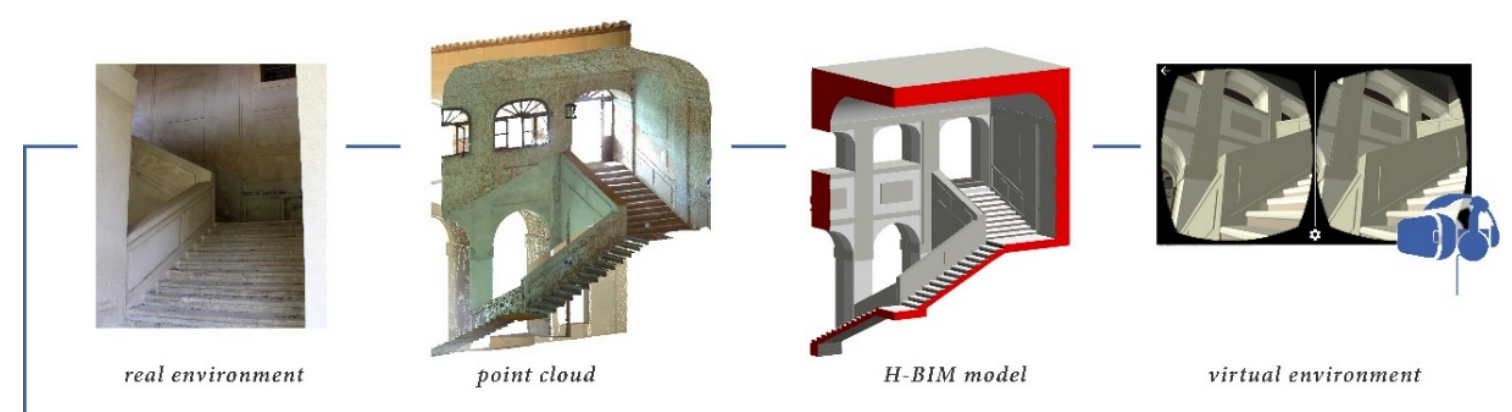

framework CLOUD-to-BIM-to-VR

Figure 1: From reality to virtual reality: project framework applied to the Simón Ruiz Hospital. 
is innovative and promising if related to virtual reconstructions for purposes that are not only informative, but also technicalscientific aimed at documenting, studying and analysing the artefact investigated.

Therefore, this paper tells the results of a research focused on the definition of new tools for understanding and knowledge of the $\mathrm{CH}$, through alternative approaches in the reading, interpretation and visualization of architectural forms, with integrated activities of processing and use of digital data (Figure 1).

\subsection{Related works}

The field of investigation within which the research moves, if on one hand it is based on consolidated procedures for recording and manipulating the different and heterogeneous information that describe an architectural artifact (di Luggo et al., 2019; López et al., 2018; Previtali and Banfi, 2018; Pulcrano et al., 2021), on the other hand it experiments the interrelation modalities of the same in a single system of use designed to facilitate sharing. This last aspect, in particular, provides interesting research ideas where the objectives that drive the experimentation are focused on the construction of digital realities that tell the real one through interactive and computerized simulations.

The use of BIM as a support for the definition of XR projects is a topic of great interest and much investigated in the scientific community of the sector (Banfi et al., 2019; Parrinello and Dell'Amico, 2021; Pybus et al., 2019; Quattrini et al., 2018). But, to date, no well-defined or consolidated methodologies have yet been achieved to manage the graphic and descriptive information relations that are established in the various operational phases.

Looking at the specific field of Virtual Reality (VR) applied to $\mathrm{CH}$, there are various topics that emerge, some of which combine theoretical issues with purely practical ones in order not to consider the H-BIM model as a mere geometric example of the artefact, but as a tool of knowledge in the VR environment. Among these, there is no doubt that the need emerges to ensure that the geometric, topological and semantic characteristics of the different smart-objects, as well as the quality and quantity of the associated metadata remain unchanged in VR. This aspect is also closely linked to the construction and computerization of the components of the 3D model on which the fruition is based. These, in fact, in traditional VR approaches are often characterized by a certain degree of uncertainty due to the simplifications introduced during the modelling phase, significantly affecting the accuracy and authenticity of the displayed data (Roussou, 2002). Otherwise, the use of the H-BIM model allows to arrive at more reliable solutions, since the application of the methodology and the operational framework presupposes the creation of a digital model coherent with reality, both in the visual aspects and logical-functional one. In this way, any inconsistencies between the geometric component and the database in the virtual environment are also limited, since the different information is linked by univocally determined relationships.

The use of the H-BIM model as part of a wider workflow, moreover, facilitates the updating operations in itinere during the development of VR - or possibly following for changes in the state of the places and for interventions -, being these contextual between 3D geometric data and alphanumeric data in the BIM platform, as well as consistently corresponded also in the VR environment.

The sharing and visualization of the information model, which is often too complex for non-expert users, is still a crucial issue. Heterogeneous and technically coded contents, as well as the inherent difficulties in the manipulation of dedicated software tools and in the identification of a specific information within the BIM database, in fact, pose important limitations that significantly affect the usability of the model. In this perspective, the $H$-BIM-to-VR process facilitates its use and accessibility while allowing, at the same time, to expand the application field of the BIM methodology also to different areas and not strictly related to those of the AECO sector.

However, to date, we still face the operational difficulties linked to one-way data flows between BIM-authoring and VR platforms and the choice of the system for the construction and execution of the same. The first aspect also recalls issues related to exchange formats, which require to operate with conversions of the information model - generally in the .obj and . $f b x$ formats so that it can be read and manipulated in the platforms dedicated to the construction of XR fruition. The second one, on the other hand, strongly affects the personalization of the VR experience not always fully guaranteed - and the ways in which it is enjoyed due to the different degree of immersion and interactivity that can be achieved.

\subsection{Case study: Simón Ruiz Hospital in Medina del Campo (Valladolid, Spain)}

The Simón Ruiz Hospital in Medina del Campo (Valladolid, Spain) (Figure 2) was chosen as a case study on which to replicate the operational phases of the research and validate the theorized process.

The complex, built between the end of the 16th and the beginning of the 17 th century, is due to both the modernization and deep transformation of the health system promoted in those centuries by the Spanish monarchy, and to the idea of the founder to create a work that remained in the memory of the place and lasted over time. In fact, leaving out the dimensions that presents - (perhaps) excessive for the time of construction and for the territorial context in which it is inserted -, the articulated functional program that characterizes it, the result of a hybridization between the traditional hospitals of the time and the convent complexes, together with the orientation that favors sunshine and natural ventilation, made it a great example of innovation (Navarro García, 1998).

Built in a strategic position on the road that once connected the city of Salamanca to Valladolid - at the very gateway to the town of Medina del Campo -, today the Hospital Simón Ruiz is located between the two most important points of Medina, Plaza de Toros and Plaza Mayor (Figure 3).

Currently, the hospital complex is in very precarious conditions due to abandonment over time and developed in a few decades. However, it is considered as a dynamization incubator for the

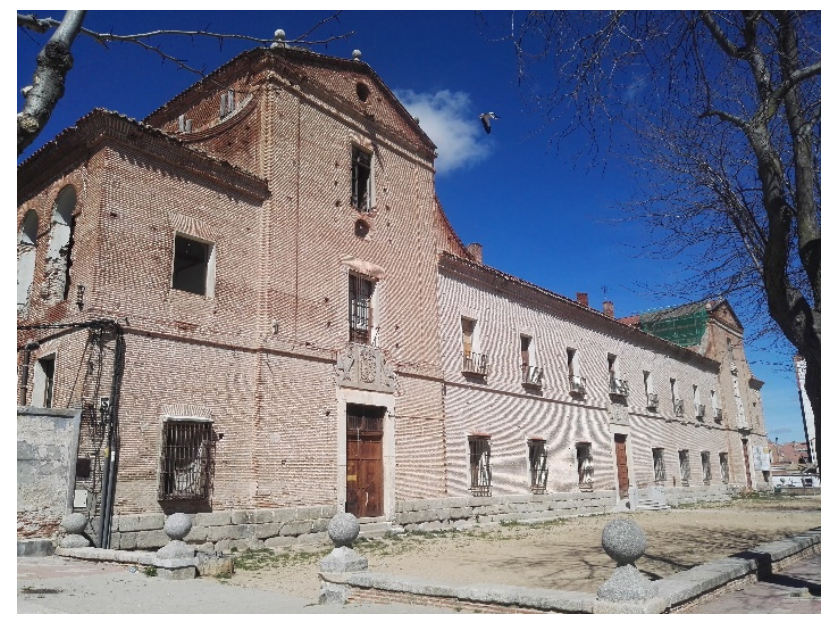

Figure 2: Simón Ruiz Hospital on Avenida Portugal 

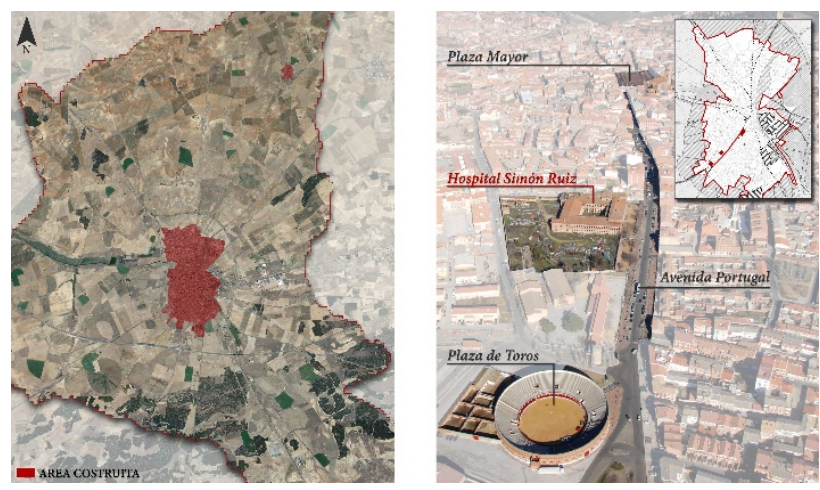

Figure 3: The Simón Ruiz Hospital in the urban context of Medina del Campo (Valladolid, Spain).

entire territory. From here move the revitalization objectives that lead the administration to recover the building by promoting various projects and activities, in order to create a real $h u b$ of cultural, social and economic growth not only for the site itself, but also for the whole territory.

\section{FROM REAL TO VIRTUAL REALITY: THE METHODOLOGICAL APPROACH}

The research focused on the different aspects connoting the connection between the products obtained from the reality-based surveys, the information modelling and the construction of VR environments, pursuing a continuous methodological approach in the collection, processing and management of data relating to the Simón Ruiz Hospital. In fact, a broad knowledge system has been built, rich in historical-informative and technical-scientific information, where the results obtained from the investigation, reading and representation of the site converge and are accessible.

Operationally, the experimentation began with bibliographic (Pulido Serrano, 2017; Sánchez del Barrio, 2016) and archive searches, to which instrumental surveys were associated in order to understand and record the morpho-geometric, metric and color information and, consequently, determine the current state of the sites.

Specifically, two survey campaigns with range-based instrumentation led to the spatial definition of a substantial part of the complex, describing the planimetric asset and the compositional, formal characteristics of the elevations. This first
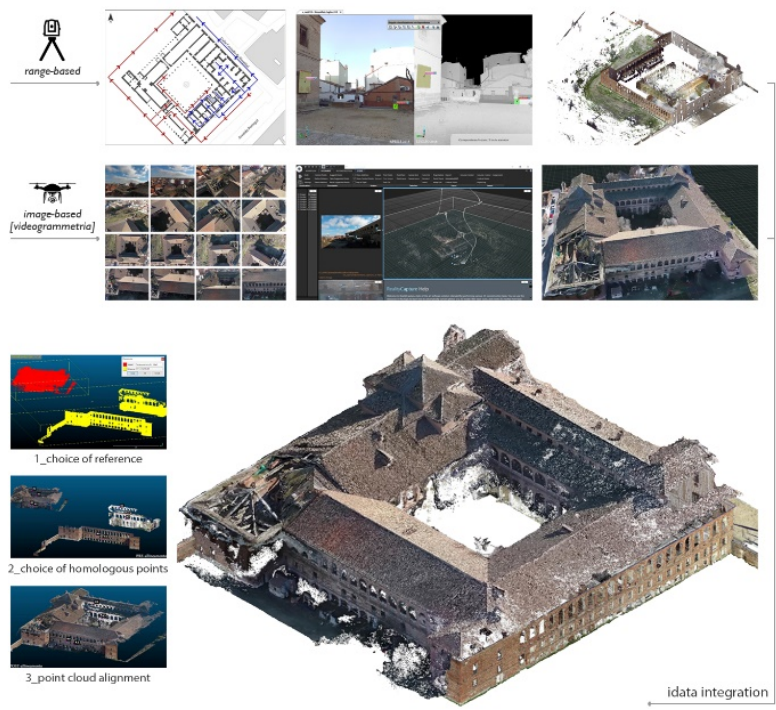

Figure 4: Point cloud of the Simón Ruiz Hospital obtained from the integration of reality-based surveys.

point cloud was later integrated with image-based processing of the videogrammetric type in order to complete the information in the missing parts. Several video acquisitions were then processed in SfM software, but the one that takes up the roofs was decisive in returning the general volume of the complex (Figure 4).

The point cloud proved to be fundamental in the construction of the H-BIM model, since from the analysis of the orthophotoplanes of the plan and elevation were derived the simplification rules to be implemented during the modelling and systemization - in the BIM environment - of the component constituents the building system of the Simón Ruiz Hospital Autodesk Revit software was chosen for research -. These rules, based on the identification of geometric rhythms and the repetitiveness of the elements, made the construction of the model quick. While the graphic and tabular abacatures, drawn up as a synthesis of the various information in the document corpus, were of support to enrich the various objects of the model with informative contents, thus making them smart. (Figure 5).

Finally, the verification of the degree of deviation between the $\mathrm{H}-\mathrm{BIM}$ model and the point cloud made it possible to validate the geometric simplification process and, where necessary, to address the appropriate corrections in order to obtain a digital data consistent with the reality it represents. In fact, a double

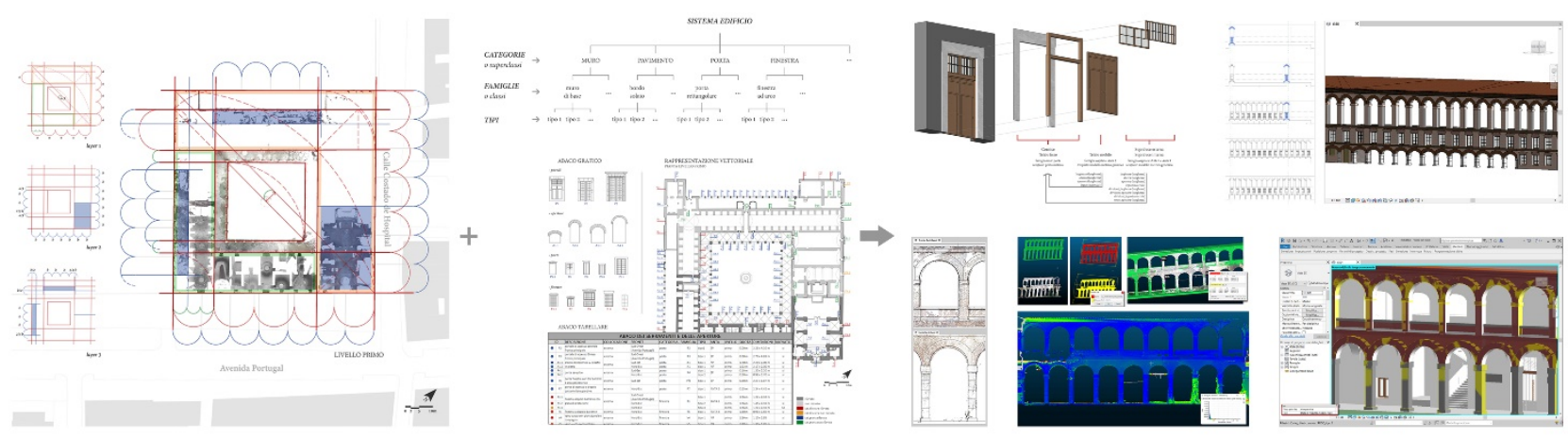

Figure 5: Synthesis of the modelling process of the Simón Ruiz Hospital. 


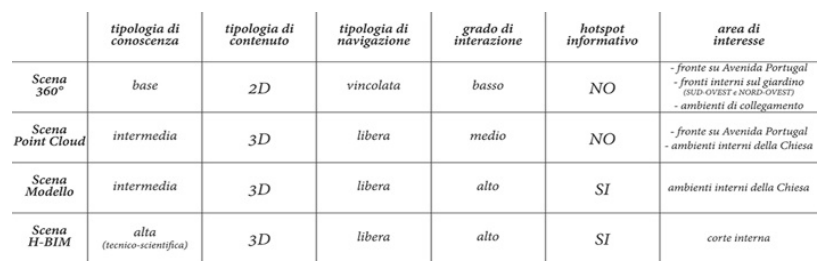

Figure 6: Comparative synthesis of the characteristics of the four scenes designed for the VR App of the Simón Ruiz Hospital.

information model has been built, on the one hand purely geometric - but which still guarantees a strong likelihood with the artefact - , on the other hand more closely related to real forms.

The H-BIM model - together with the point clouds - was used as a three-dimensional support for VR. This last phase was characterized by theoretical reflections and practical-application activities aimed first at the conversion of the different models into compatible formats and, later, at the design and programming of the App. Several tests were performed for the definition of the operational sequences suitable for the construction of the VR, so that the results obtained were coherent with the set objectives, linked not only to the navigation of the geometric component, but also to the visualization of the metadata for each of the smartobjects of which is composed and, more generally, to the systemization in a single tool of the different types of information concerning the Medinense hospital complex.

Consequently, the key points addressed concerned, in particular, (i) the methods of converting the H-BIM model - geometry and metadata - so that it could be viewed and manipulated in VR systems, (ii) the design and organization of the contents and execution of the VR and, finally, (iii) the planning and development of the fruition project.

\section{RESULTS: THE VR APP}

The final result achieved through the framework used is characterized by the VR App for mobile devices that relates, according to different contents, degrees of in-depth study and navigation methods, the information acquired - or elaborate during the multiple phases that involved the research whit alternative ways and completely independent from the software that generated them.

A system was therefore structured a multidimensional and multimodal fruition path of the investigated artefact. The characteristics that connote it are related to the setting of incremental levels of knowledge, different by type of content displayed (historical, descriptive, technical, etc.), form in which it is presented (spherical and photographic images, point cloud, geometric and informative model, textual descriptions, etc.) and integration of hotspots for extend the informative and descriptive content. (Figure 6).

Preliminary to the development of the App was the definition of the fruition system, intended both as devices to be used to allow a certain degree of immersion in the digital scenes and interactivity with the objects present in them, and as software for its real programming. It is clear that the choice of the various components arose from the analysis of the potential and criticalities of each of them, from the evaluation of the results they allow to obtain, but also from reflections on the diffusion of the system so that it can be used by a greater number of users. This last aspect, in particular, has directed towards a low-cost

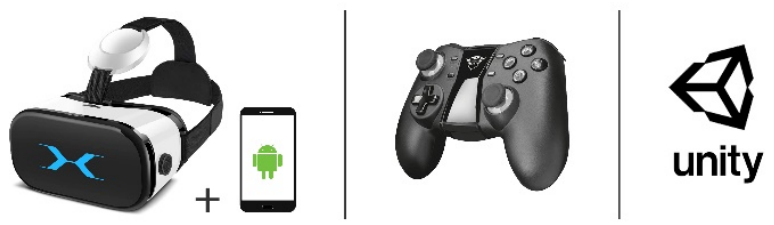

Figure 7: Definition of the technological system for the programming, execution and management of the VR experience.

system (Figure 7), identifying in the smartphone - now fully in daily use - the device suitable for carrying out the VR use.

Specifically, therefore, it was decided to set up an App managed by Android operating systems. Cardboard-type display media simulate the three-dimensionality and total immersion of the different levels; while traditional game controllers - suitably customizable in executable commands - allow user navigation without pre-established schemes and interaction with the contents presents in the App.

Everything was programmed using the Unity $3 D$ open source gaming engine software, deemed suitable to meet the set objectives. In fact, unlike the viewers directly associated with the BIM-authoring platforms - specific tools and plugins for VR use of the information model -, Unity $3 D$ allows extensive customization during VR programming, being able to intervene on various technical aspects (actions allowed in levels, insertion of objects or characters to interact with, etc.) and graphics (rendering of textures, setting of light sources, etc.).

Furthermore, being a multi-platform software, with small corrections it is possible to adapt the VR project for various instruments (smartphones, tablets, PCs, etc.) and operating systems (Android, IOS, Windows, etc.), thus making it replicable on different media according to the needs and adaptable both for on-site and remote and web-based use.

\subsection{The VR App scenes}

The application built for the Simón Ruiz Hospital is divided into four levels - or scenes in Unity $3 D$ logic - interrelated by an initial menu (Figure 8). The methods of execution vary according to the contents inserted, while the degree of interaction depends

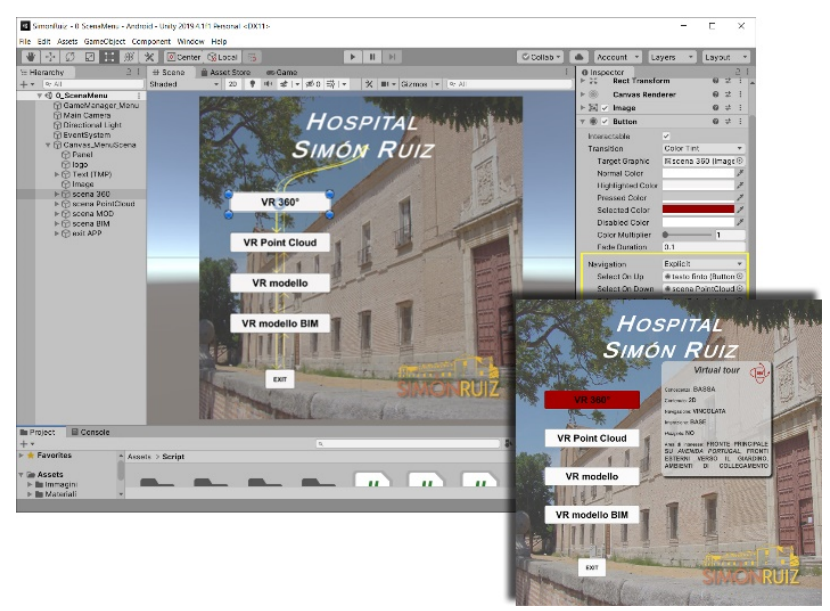

Figure 8: Start menu of the VR App. Selection of the key corresponding to the $360^{\circ}$ Scene and display of the related graphic and textual descriptions. 

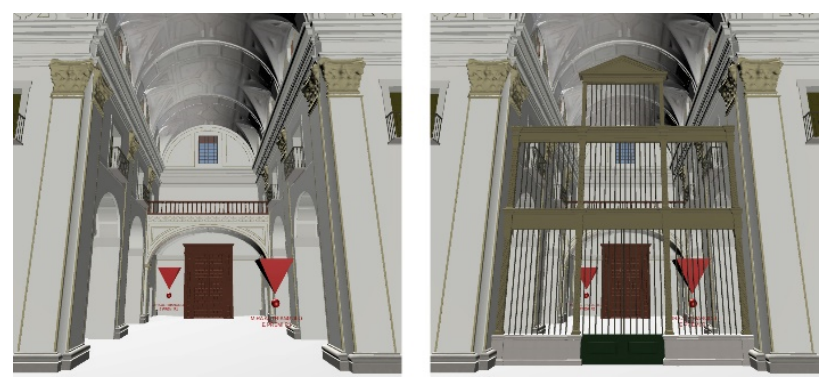

Figure 9: Activation of the on/off functions connected to the hotspot that allows you to manage the display of the gate. View of the nave from the transept.

on the narrative structures set for the specific scene. What changes are not only the activities that the user can perform and the methods of execution of the VR, but also the type of object with which he relates.

3.1.1 Main menu: The menu represents the initial scene displayed when the App is started and was designed as a support element to guide and orient the user in choosing the environment to be tested, described by means of graphic and textual summaries. In addition of the menu structure there are several elements that are not necessary for the correct execution of the menu, but contribute to complete its graphics appearance.

The operating procedure carried out for the construction of the main menu has been partially replicated in the context menus of the various scenes. These, however, differ from it for the purposes for which they were designed - activation of specific actions and/or variations of the settings -, for the elements that characterize them and for the activation mode - their display takes place using the appropriate button on the controller-.

3.1.2 $360^{\circ}$ scene: The first level corresponds to the zero level of the App, that is to a simplified, immersive but not at all interactive fruition and without additional content beyond those displayed. As happens in the more traditional virtual tours, the fruition is entrusted to the visualization of spherical images and, therefore, bound to predetermined points of view, which the user can change using the buttons on the controller.

The different images that make up the scene have been extrapolated from the data acquired during the range-based surveys and show the hospital complex in its real consistency, marked by the signs of time and without filters that alter its perception. Their equirectangular projection has been appropriately edited in order to make it respond to the 1:2 ratio, necessary for correct stereoscopic visualization. This takes place through a virtual camera that simulates the user's view allowing him, as is well known, to feel the spatiality of the scene through the rendering of the same portion from two slightly different points of view.

3.1.3 Point Cloud scene: This scene is similar to the previous one as far as the displayed contents are concerned, but it differs from it for the type of the same and for the methods of use. Here, in fact, the user navigates freely within the reality-based point clouds, by operating the directional arrows and the analog sticks of the controller.

In order for the survey data to be manageable and manipulable in the Unity $3 D$ environment, polygonal models have been reconstructed starting from the point clouds of the different portions of Medinense hospital. The creation of the mesh has seen several operational stages of editing, necessary to obtain an object with a high photorealistic yield but characterized by a not

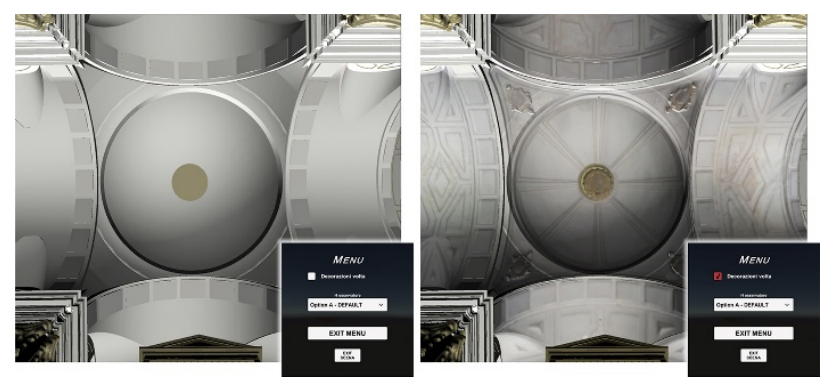

Figure 10: Activation and deactivation of the decorative components of the vaults through the context menu of the Model scene.

excessively high number of polygons. This has concurred not to burden excessively on the graphical component of the system used for the fruition, avoiding eventual delays in the rendering of the same models and, therefore, slowdowns in the execution of the VR.

3.1.4 Model scene: The elements that make up the Model scene are made up of the H-BIM model - only in the geometric component - and various hotspots. The choice to limit the type of data that can be deduced from the information model was dictated by the desire to set up a purely informative and nontechnical-scientific scene.

The contents implemented in it also move in this direction. It should be noted, however, that not all the hotspots inserted are dedicated to providing additional information and, consequently, to increasing the contents of the VR. Some, in fact, are intended to induce a variation in the view so that the user can feel the space in a different way from the real one or according to another perspective (Figure 9).

In addition to the hotspots, the Model scene includes all those decorative elements (capitals, molded cornices, etc.) that in the BIM environment have not been modeled but inserted directly as portions of the point cloud. Some of them, like the vault decorations, can be activated/deactivated from the contextual menu of the scene (Figure 10).

3.1.5 BIM scene: The last scene that makes up the Simón Ruiz Hospital App is set on the navigation of the H-BIM model and on the ability to view the metadata (Figure 11) associated with each smart-objects in Autodesk Revit.

To this initial information, additional contents have been implemented that can be activated both by the information hotspots affixed to the scene and from the contextual menu of the same. Specifically, it was considered interesting to insert thematic cards, detailed drawings, synoptic tables, etc., or rather, different documents which, extrapolated from the information model in order not to lose coherence in the data represented, have been converted into special formats and imported into the VR environment. While other information, such as the display of the parts taken exclusively from the reality-based surveys or the geometric rules and proportional relationships underlying the composition of the fronts, can be activated/deactivated from the contextual menu.

Therefore, if on the one hand the BIM scene represents the synthesis of all the technical solutions - detailed in the following point - used in the previous levels, on the other hand it differs from it for the purely technical aspect that characterizes it. It was in fact conceived and programmed as a support for the specialists of the AECO sector, in order to provide a useful tool to allow a first orientation approach to the information model. However, the simplicity and immediacy in obtaining the different contents 

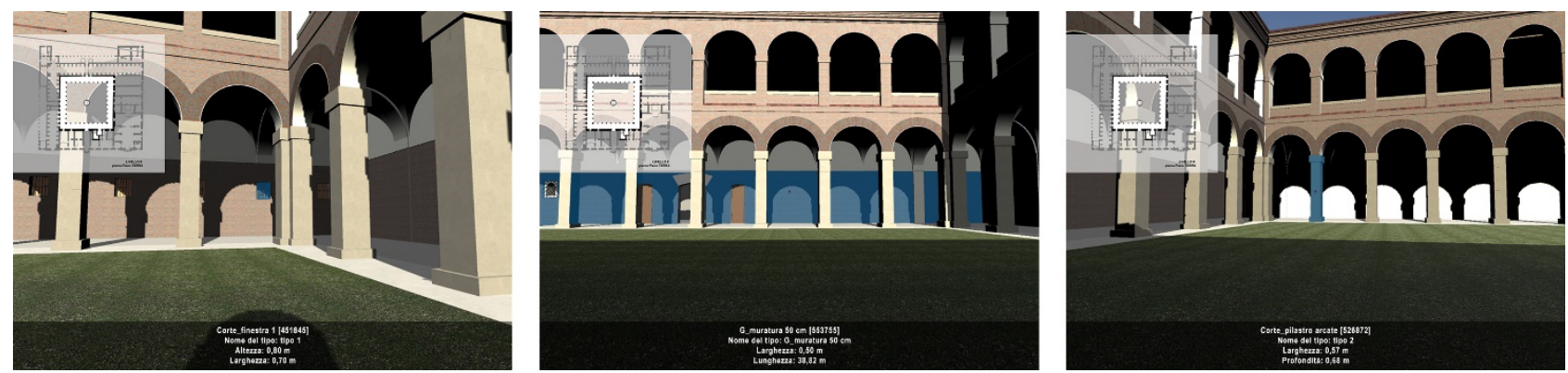

Figure 11: H-BIM scene: smart-objects selection (in blue) and visualization of related metadata in VR environment.

inserted makes it usable by a wider target of users, interested or intrigued by understanding, for example, the specific characteristics of the elements of the complex or the simplification choices adopted in the construction of the H-BIM model.

\subsection{The technical solutions used in the construction of the VR App}

What was described in the previous point was achieved through a timely organization and programming of operational solutions, and with the use of specific tools.

In particular, the first aspect on which the research focused was the definition of the connection methods between the H-BIM model and the workspace intended for VR programming. It was therefore necessary to identify a process of converting the Revit file that met the purposes and allowed to correctly manage the data in Unity $3 D$. By examining through the different and possible feasible solutions and comparing them with the results they allow to obtain, it was decided to experiment with the Export to Unity add-on which, differently from other existing applications, allows you to customize the export and set, among the provided settings, that the information model maintains the various properties attributed to the smart-objects that compose it. The latter are encoded in a dedicated programming script and are then re-associated with the geometric component in the game engine software through the ID of the element which, therefore, is corresponding between the BIM environment, the VR one and the script itself (Figure 12).
Having ascertained the outcome of the conversion, both of the HBIM model and of the related metadata, the following operation involved their fruition in VR, that is, as a user, accesses the various information. This aspect is clearly linked to the execution of the action, which first requires the selection of the digital object and then its recognition, the reading of the ID that distinguishes it and the display of the various data on the smartphone screen. In virtual fruition systems, the selection can take place through pointing devices, using eye tracking or through specially designed and programmed solutions. The choice, as is well known, is highly dependent on the utilization system fruition. In the specific case of the Simón Ruiz Hospital, the Raycasting technique, associated with the functions set for the controller keys and text recognition codes (tags), proved to be the choice that best meets the purposes. Conceptually, this allows you to simulate the trajectory of the user's view using invisible rays which, hitting the objects in the scene, provide the information - or activate the functions - associated with them. The various operational phases that characterize it have therefore been defined and the scripts necessary for its execution have been programmed.

The Raycasting technique was applied both for the smart-objects of the model and for the actions associated with the hotspots in the Model and BIM scenes. The firsts, however, are characterized by a double level of recognition - based on the tag and the ID while the latter are related to unique tags.

The movement of the user in the different scenes of the App was managed by building a specific object, a player (Figure 13), and set to be a walk-through first person. The player was accompanied by various information, including scripts that allow
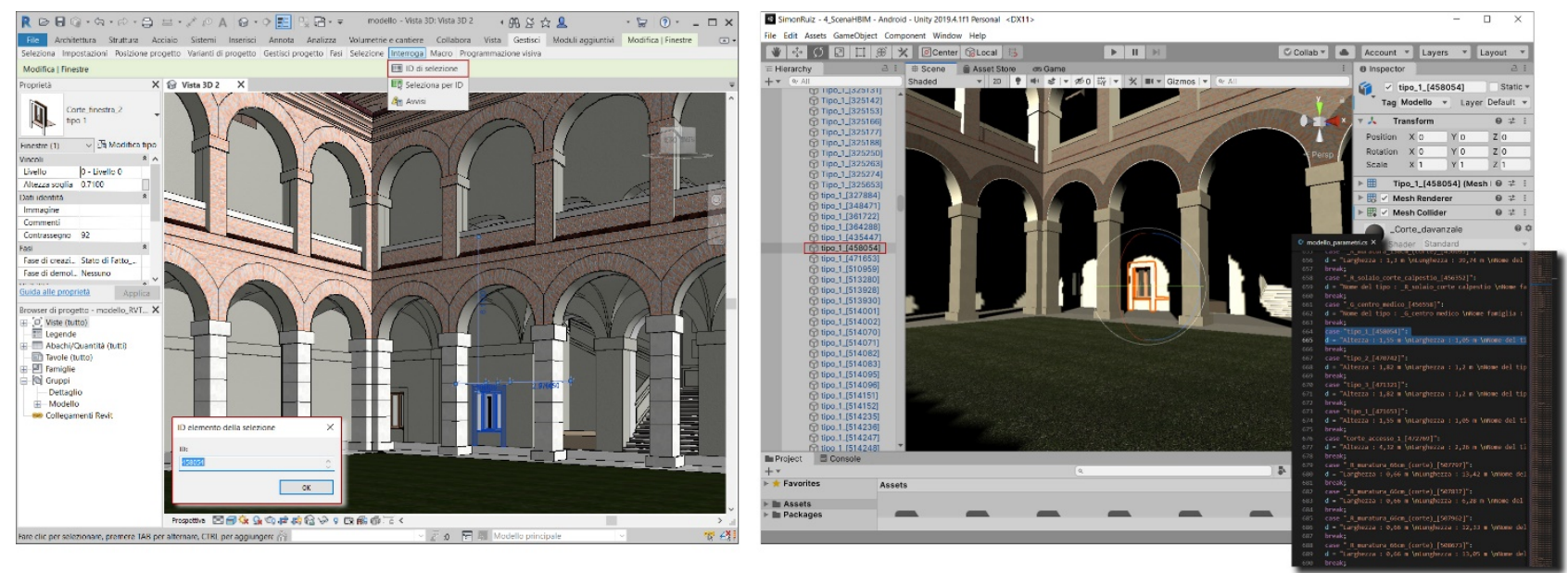

Figure 12: Correspondence in the ID code of the smart-objects read in Autodesk Revit, of the same object in Unity 3D and of the relative line of code in the parameter script. 


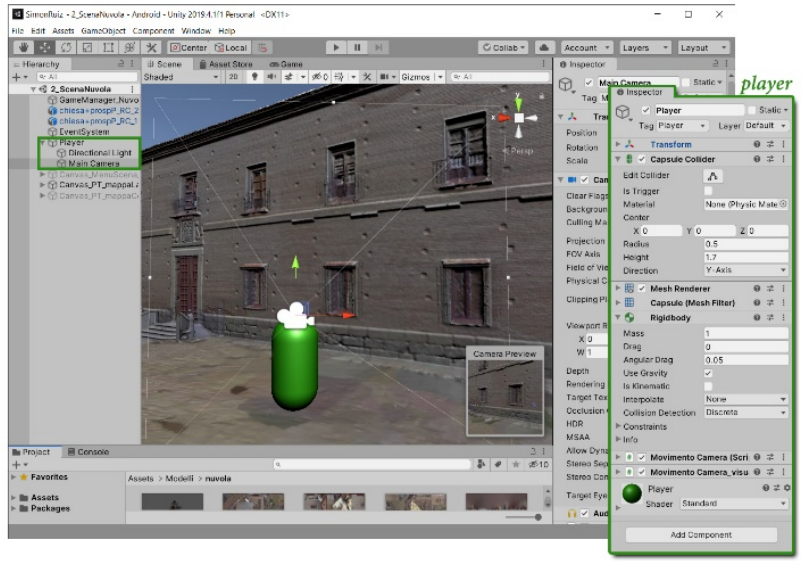

Figure 13: Construction of the player and display of the different components that characterize it.

you to relate motion outputs to the inputs that the user induces on the scene through the controller so that he has the real sensation of walking through the scene he is enjoying. The player has also been associated with the virtual camera that can be oriented in the right/left and up/down directions in relation to the user's view, which is determined by the sensors present in the device used to run the App.

\section{CONCLUSION}

The experimentation conducted is part of the current scientific debate by addressing, among the various topics investigated, the usability of the information model. Various, in fact, are the applications and research still in progress for which the BIM/HBIM model is seen as an intermediate phase of a broader workflow. In particular, the need to conform new tools of understanding and knowledge, which are explanatory of the information continuum - both graphic and descriptive - that is generated in the investigations on $\mathrm{CH}$, has prompted the present study to identify an alternative framework in reading, interpretation and visualization of architectural forms, relating informative and technical-scientific aspects in a single integrated process of elaboration and use of digital data.

The contribution therefore tells the specific point of view used in addressing the issue in question and although the proposed solutions are strongly centered on the Medinense hospital, the identified framework can still be reiterated, directing the application choices in relation to the particularities that from time to time once the investigated artifact presents.

In conclusion, the App designed is configured as a useful tool to facilitate the management and programming of interventions on the building, becoming a user-friendly guide in understanding the complex information structure that characterizes the model, which can always be implemented and updated with semiautomated processes, which correspond to modifications of the BIM data, variations of the VR. Furthermore, it makes it usable in alternative ways, overcoming the temporary difficulties of accessibility and promoting its disclosure and dissemination even beyond the territory in which it is located.

\section{REFERENCES}

Banfi, F., Brumana, R., and Stanga, C., 2019. Extended Reality and Informative models for the Architectural Heritage: from
scan-to-BIM process to Virtual and Augmented Reality. Virtual Archeology Review, Universidad Politecnica de Valencia, vol. 10, n. 21, pp. 17-30. https://doi.org/10.4995/var.2019.11923

Di Luggo, A., Palomba, D., Pulcrano, M., and Scandurra, S., 2019a. Theoretical and Methodological implication in the Information Modelling of Architectural Heritage. Bolognesi, C., and Santagati, C. (eds), Impact of Industry 4.0 on Architecture and Cultural Heritage, edizioni IGI Global, IGI Global, pp. 2048. DOI: 10.4018/978-1-7998-1234-0.ch002.

López, F. J., Lerones, P. M., Llamas, J., Gómez-García-Bermejo, J., and Zalama, E., 2018. A Review of Heritage Building Information Modeling (H-BIM). Multimodal Technologies and Interaction, vol. 2(2):21. https://doi.org/10.3390/mti2020021

Navarro García, L., 1998. El Hospital General de Simón Ruiz en Medina del Campo. Fábrica e idea. Junta de Castilla y Leon, Consejer!a de educacion y cultura.

Parrinello, S., and Dell'Amico, A., 2021. From Survey to Parametric Models: HBIM Systems for Enrichment of Cultural Heritage Management. Bolognesi, C., and Villa, D. (eds), From Building Information Modeling to Mixed Reality, Springer, pp. 89-107. https://doi.org/10.1007/978-3-030-49278-6_6

Previtali, M., and Banfi, F., 2018. Towards the Definition of Workflows for Automation in HBIM Generation. Ioannides, M., Fink, E., Brumana, R., Patias, P., Doulamis, A., Martins, J., and Wallace, M. (eds), Digital Heritage. Progress in Cultural Heritage: Documentation, Preservation, and Protection. EuroMed 2018. Lecture Notes in Computer Science, Springer, Cham, vol. 11196, pp. 52-63. https://doi.org/10.1007/978-3-03001762-0_5

Pybus, C., Graham, K., Doherty, J., Arellano, N., and Fai, S., 2019. New realities for Canada's Parliament: a workflow for preparing Heritage BIM for Game Engines and Virtual Reality. The Int. Arch. Photogramm. Remote Sens. Spatial Inf. Sci., Vol. XLII-2/W15, pp. 945-952. https://doi.org/10.5194/isprsarchives-XLII-2-W15-945-2019

Pulcrano, M., Scandurra, S., Fragalà, E., Palomba, D., and di Luggo, A., 2021. Multi-sensor data acquisition and integration processes for the study and documentation of the Church of Santa Maria degli Angeli in Pizzofalcone in Naples. The International Archives of the Photogrammetry, Remote Sensing and Spatial Information Sciences, vol. XLVI-M-1-2021, pp. 571-578. https://doi.org/10.5194/isprs-archives-XLVI-M-1-2021-5712021

Pulido Serrano, J.I. (eds), 2017. Más que negocios Simón Ruiz un banquero español del siglo XVI entre la penínsulas ibérica e italiana. Iberoamericana Editorial Vervuert.

Quattrini, R., Battini, C., and Mammoli, R., 2018. HBIM to VR. Semantic awareness and data enrichment interoperability for parametric libraries of Historica Architecture. The International Archives of the Photogrammetry, Remote Sensing and Spatial Information Sciences, vol. XLII-2, pp. 937-943. https://doi.org/10.5194/isprs-archives-XLII-2-937-2018

Roussou, M., 2002. Virtual Heritage: from the reserch lab to the broad pubblic, in Virtual Archeology. Nicolucci F. (eds), Proceedings of the VAST Euroconferenze, Archeopressa, pp. 93100.

Sánchez del Barrio, A. (eds), 2016. Simón Ruiz mercader, banquero y fundador. Fundación Museo de las Ferias. 